\title{
Ultrasound promoted synthesis of chromeno[2,3-b]pyridines and their evaluation as lipid peroxidation inhibitors
}

\author{
Eleni Dimitriadou, ${ }^{a}$ Marianna Raftopoulou, ${ }^{\text {a }}$ Paraskevi M. Kasapidou, ${ }^{a}$ Constantinos A.

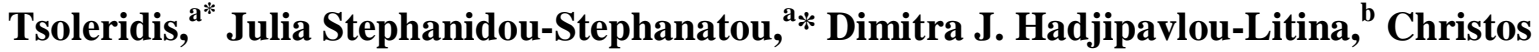 \\ Kontogiorgis, ${ }^{b, c}$ Agathi Pritsa, ${ }^{c}$ and Athanasios Papadopoulos ${ }^{c}$ \\ ${ }^{a}$ Laboratory of Organic Chemistry, Department of Chemistry, Aristotle University of Thessaloniki, \\ Thessaloniki 54124, Macedonia, Greece \\ ${ }^{b}$ Department of Pharmaceutical Chemistry, School of Pharmacy, Aristotle University of \\ Thessaloniki, Thessaloniki 54124, Macedonia, Greece \\ ${ }^{c}$ Department of Human Nutrition \& Dietetics, Alexander Technological Educational Institute, PO \\ Box 141, Thessaloniki 57400, Macedonia, Greece \\ E-Mail: tsolerid@chem.auth.gr, ioulia@chem.auth.gr
}

DOI: http://dx.doi.org/10.3998/ark.5550190.0015.500

\begin{abstract}
A series of 2,3-disubstituted-5-oxochromeno[2,3-b]pyridine derivatives were synthesized under ultrasound irradiation, and also under conventional methods, from the reaction of 3cyanochromones with some active methylene compounds in the presence of a stoichiometric base. The structures of the products were unambiguously elucidated by 1D and 2D NMR experiments. Full assignment of all ${ }^{1} \mathrm{H}$ and ${ }^{13} \mathrm{C}$ NMR chemical shifts has been achieved. In addition, the lipid peroxidation inhibition of the synthesized chromenopyridine derivatives was evaluated.
\end{abstract}

Keywords: Antioxidant activity, chromeno[2,3-b]pyridines, 3-cyanochromones, lipid peroxidation inhibition, sonochemistry

\section{Introduction}

In recent decades, ultrasound irradiation has been used more and more frequently in organic synthesis. ${ }^{1,2}$ The use of ultrasound to accelerate reactions has proven to be a particularly important tool for meeting the Green Chemistry goals of minimization of waste and reduction of energy requirements. ${ }^{3,4}$ Applications of ultrasonic irradiation are playing an increasing role in chemical processes, especially in cases where classical methods require drastic conditions or prolonged reaction times. The excellent review of Cella and Stefani ${ }^{5}$ clearly showed the importance of taking advantage of the unique features of ultrasound-assisted reactions to synthesize heterocyclic ring systems. 
Heterocyclic compounds are highly ranked among pharmaceutically important natural and synthetic materials. The remarkable ability of heterocyclic nuclei to serve both as biomimetics and active pharmacophores has largely contributed to their unique value as traditional key elements of numerous drugs. As a result, in the last years the preparation of natural-product-like libraries has become a foremost strategy for the discovery of new drugs. ${ }^{6}$ In addition, merging privileged structures constitutes a powerful strategy to reach drug-like bioactive compounds in a fast and productive manner. ${ }^{7}$ In this context, the discovery of core structures having this potential is one of the main challenges for the pharmaceutical industry in its search for rich sources of new molecular entities. $^{8}$

In this way, the chromenopyridine scaffold may be regarded as the fusion of two main fragments: of a chromene and of a pyridine moiety. Both fragments display a wide range of biological activities; they are present in many drugs and have been extensively studied. Chromenes form a group of either naturally or synthetically obtained compounds possessing a broad diversity of biological activities with an impact in the treatment of inflammation and ulcers, ${ }^{9}$ schizophrenia, ${ }^{10}$ cancer, ${ }^{11}$ etc.

On the other hand, pyridines are ubiquitous in nature and pivotal in synthetic and medicinal chemistry constituting a useful core for biomedical research. ${ }^{12}$

Concerning chromenopyridines, a wide array of biological activities has been described for compounds containing this core in their structures. This small, compact scaffold is rich in intermolecular interactions, and can be easily functionalized and diversified. Owing to such interesting properties, the medicinal chemistry around this structural class is emerging as an active subject of research, not only in academia, but especially in the pharmaceutical industry. In this context, some chromenopyridines have been reported to be alpha adrenergic antagonists, ${ }^{13}$ potent bronchodilating agents, ${ }^{14}$ and useful in the treatment of bronchitis and asthma. ${ }^{15}$

In view of our continuous interest in merging privileged structures containing a chromene moiety ${ }^{16-18}$ we decided to pursue a more detailed investigation concerning the synthesis of chromeno[2,3-b]pyridines from the reaction of 3-cyanochromones with active methylene compounds in the presence of a stoichiometric base.

\section{Results and Discussion}

\section{Chemistry}

Some early reports ${ }^{19-21}$ concerning the synthesis of chromeno[2,3-b]pyridines from 3cyanochromones with some active methylene compounds by refluxing in ethanol in the presence of piperidine are reported in the literature. In addition recently, the reaction between cyanochromones with some active methyl and methylene compounds was studied in the presence of DBU, as a base, whereupon chromenopyridines were isolated in satisfactory yields. ${ }^{22}$ In the present study we decided to pursue an investigation concerning the possibility of improving the reaction yield by using Green Chemistry conditions, namely ultrasound irradiation, and to provide a structure elucidation based on detailed NMR study since the proposed in the literature structure of chromeno[2,3-b]pyridines ${ }^{19-22}$ was based on inadequate ${ }^{1} \mathrm{H}-\mathrm{NMR}$ data. Thus initially, 3- 
cyanochromone, dimethyl acetonedicarboxylate and p-toluidine were refluxed in ethanol for $4 \mathrm{~h}$, whereupon the yellow solid, which was formed upon cooling of the reaction mixture, was filtered off and recrystallized from ethanol to give the chromenopyridine 3a in $60 \%$ yield. The reaction was repeated with other bases and the results are given in Table 1.

Table 1. The effect of different bases on the yield of product 3a

\begin{tabular}{ccc}
\hline Entry & Base & Yield (\%) \\
\hline 1 & $p$-toluidine & 60 \\
2 & $p$-chloroaniline & 45 \\
3 & DMAP & 35 \\
4 & DABCO & 68 \\
5 & DBU & unidentified \\
& & product \\
6 & cinchonine & 47 \\
7 & DABCO with & 85 \\
& ultrasound & \\
& irradiation & \\
\hline
\end{tabular}

Table 2. Synthesis of chromenopyridines 3<smiles>[R]OC(=O)CC(=O)CC([R20])[R20]</smiles><smiles>[R]OC(=O)Cc1cc2c(=O)c3cc([R])cc([R])c3oc2nc1C(=O)O[R]</smiles>

\begin{tabular}{cccccccccc}
\hline Entry & $\mathrm{R}^{1}$ & $\mathrm{R}^{2}$ & $\mathrm{R}^{3}$ & Product & $\begin{array}{c}\text { Yield }(\%) \\
\text { ultrasound } \\
\text { irradiation } \\
10 \text { min }\end{array}$ & $\begin{array}{c}\text { Yield }(\%)^{\mathrm{a}} \\
\text { ethanol } \\
\text { reflux }\end{array}$ & $\begin{array}{c}\text { Yield } \\
(\%)^{\mathrm{b}}\end{array}$ & $\mathrm{mp}\left({ }^{\circ} \mathrm{C}\right)^{\mathrm{a}}$ & $\left.\mathrm{mp}^{\circ} \mathrm{C}\right)^{\mathrm{b}}$ \\
\hline 1 & $\mathrm{O}$ & $\mathrm{H}$ & $\mathrm{Me}$ & $\mathbf{3 a}$ & 85 & 68 & 49 & $170-172$ & $168-169$ \\
2 & $\mathrm{H}$ & $\mathrm{H}$ & $\mathrm{Et}$ & $\mathbf{3 b}$ & 82 & 65 & - & $153-155$ & - \\
3 & $\mathrm{Me}$ & $\mathrm{H}$ & $\mathrm{Me}$ & $\mathbf{3 c}$ & 80 & 56 & - & $150-151$ & - \\
4 & $\mathrm{Me}$ & $\mathrm{H}$ & $\mathrm{Et}$ & $\mathbf{3 d}$ & 81 & 67 & - & $131-133$ & - \\
5 & $\mathrm{Me}$ & $\mathrm{Me}$ & $\mathrm{Me}$ & $\mathbf{3 e}$ & 86 & 68 & 58 & $175-177$ & $197-198$ \\
6 & $\mathrm{Cl}$ & $\mathrm{H}$ & $\mathrm{Me}$ & $\mathbf{3 f}$ & 78 & 63 & 36 & $188-190$ & $192-193$ \\
7 & $\mathrm{Cl}$ & $\mathrm{H}$ & $\mathrm{Et}$ & $\mathbf{3 g}$ & 86 & 65 & 65 & $144-146$ & $148-149$ \\
8 & $\mathrm{Cl}$ & $\mathrm{Cl}$ & $\mathrm{Me}$ & $\mathbf{3 h}$ & 79 & 57 & - & $166-168$ & - \\
\hline
\end{tabular}

${ }^{\mathrm{a}}$ Isolated product in this work. ${ }^{\mathrm{b}}$ Ref. 21. 
In all cases chromenopyridine 3a was isolated in varying yields with exception the reaction with DBU, where most probably a three component unidentified product incorporating DBU was isolated. Finally, the reaction was investigated under ultrasound irradiation, using DABCO as a base, since DABCO provided the best results under conventional heating. Analytically, the reaction mixture in a small amount of ethanol was subjected to ultrasound irradiation for 10 min, whereupon after initial solvation of the reactants the chromenopyridine 3a was crystallized out. After the reaction conditions were optimized, the generality of the reaction was investigated with a variety of cyanochromones, with methyl and ethyl acetonedicarboxylates, and the results summarized in Table 2 .

Next, the reaction was investigated by using ethyl acetylacetate and ethyl benzoylacetate and it was found, that in order for the reaction to proceed, the stronger base DBU was necessary. No reaction was observed when $p$-toluidine was used as a base. To the contrary, concerning ultrasound irradiation $\mathrm{DABCO}$ was found to be the best base. The results are presented in Table 3.

Table 3. Synthesis of chromenopyridines 5

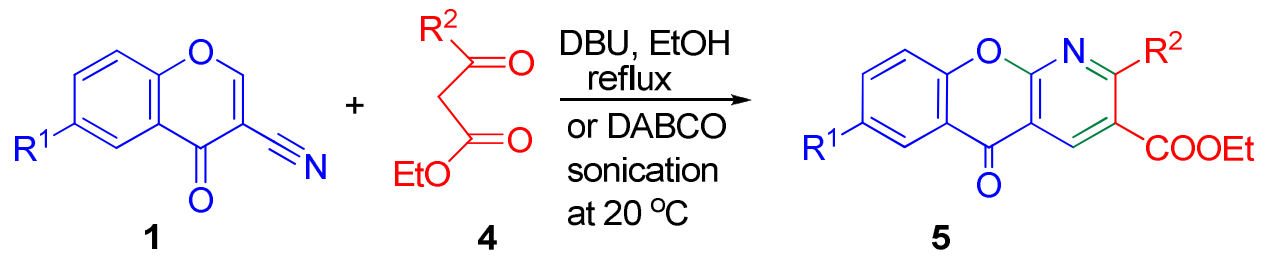

\begin{tabular}{ccccccccc}
\hline Entry & $\mathrm{R}^{1}$ & $\mathrm{R}^{2}$ & Product & $\begin{array}{c}\text { Yield (\%) } \\
\text { ultrasound } \\
\text { irradiation } \\
\text { DABCO }\end{array}$ & $\begin{array}{c}\text { Yield }(\%)^{\mathrm{a}} \\
\text { ethanol } \\
\text { reflux } \\
\text { DBU }\end{array}$ & $\mathrm{mp}\left({ }^{\circ} \mathrm{C}\right)$ & Yield (\%) & $\mathrm{mp}\left({ }^{\circ} \mathrm{C}\right)$ \\
\hline 1 & $\mathrm{H}$ & $\mathrm{Me}$ & $\mathbf{5 a}$ & $76(40 \mathrm{~min})$ & 47 & $161-163$ & $63^{\mathrm{b}}$ & $162^{\mathrm{b}}$ \\
2 & $\mathrm{H}$ & $\mathrm{Ph}$ & $\mathbf{5 b}$ & $70(15 \mathrm{~min})^{\mathrm{c}}$ & 55 & $146-148$ & $41^{\mathrm{d}}$ & $270^{\mathrm{d}}$ \\
3 & $\mathrm{Me}$ & $\mathrm{Me}$ & $\mathbf{5 c}$ & $78(35 \mathrm{~min})$ & 55 & $165-167$ & $63^{\mathrm{b}}$ & $170^{\mathrm{b}}$ \\
4 & $\mathrm{Me}$ & $\mathrm{Ph}$ & $\mathbf{5 d}$ & $72(15 \mathrm{~min})$ & 57 & $174-176$ & - & - \\
5 & $\mathrm{Cl}$ & $\mathrm{Me}$ & $\mathbf{5 e}$ & $76(40 \mathrm{~min})$ & 53 & $166-168$ & $65^{\mathrm{b}}$ & $169^{\mathrm{b}}$ \\
6 & $\mathrm{Cl}$ & $\mathrm{Ph}$ & $\mathbf{5 f}$ & $76(20 \mathrm{~min})$ & 59 & $167-169$ & - & - \\
\hline
\end{tabular}

${ }^{\mathrm{a}}$ This work. ${ }^{\mathrm{b}}$ Ref. 19. ${ }^{\mathrm{c}} 16 \%$ yield when DBU was used. ${ }^{\mathrm{d}}$ Ref. 22.

Concerning the reaction mechanism, it is conceivable that the initial attack of the anion $\mathbf{6}$, formed from the active methylene group compound $\mathbf{2}$ (or $\mathbf{4}$ ), to $\mathrm{C} 2$ of cyanochromone $\mathbf{1}$ is accompanied by the chromone ring opening to intermediate 8. After rotation around the $\mathrm{C} 3-\mathrm{C} 4$ bond and recyclization through attack of the pyran oxygen anion to the cyano-carbon C5 intermediate $\mathbf{9}$ is formed, which subsequently undergoes a new cyclo-condensation to furnish 10. Finally, after loss of water, the chromenopyridine $\mathbf{3}$ (or $\mathbf{5}$ ) is formed (Scheme 1). For the isolated products structure 11, depicted in Figure 2, could also be proposed. However, this structure was excluded on the basis 
of rigorous spectroscopic analysis (mainly 2D NMR spectra) as described in the following session. Moreover, minor products with a structure analogous to $\mathbf{1 1}$ were not identified.

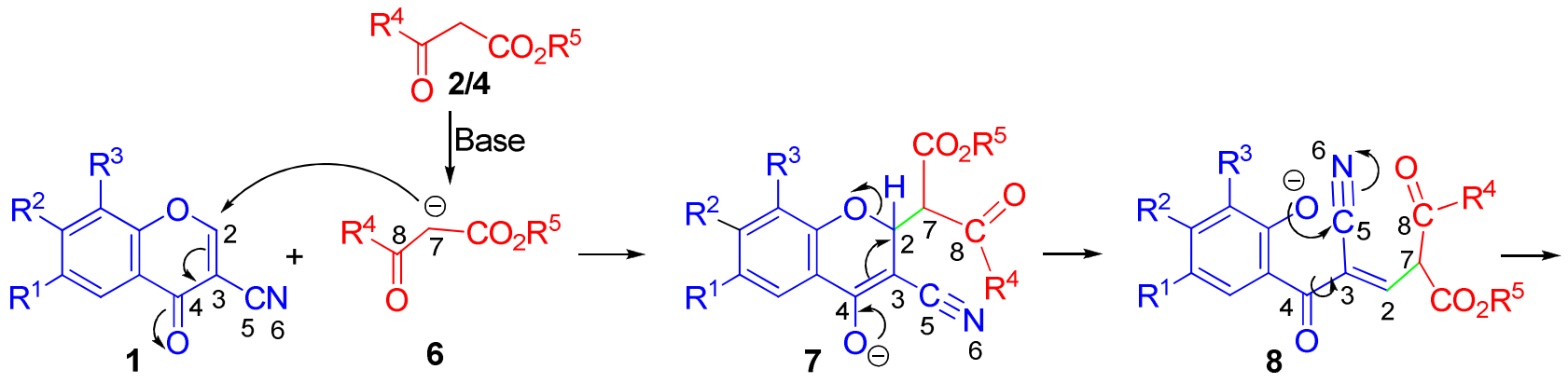<smiles>[R]c1cc2c(c([R])c1[R])OC(=O)C(=CC(=O)C([R])(C)C(=O)O)C2=O</smiles>

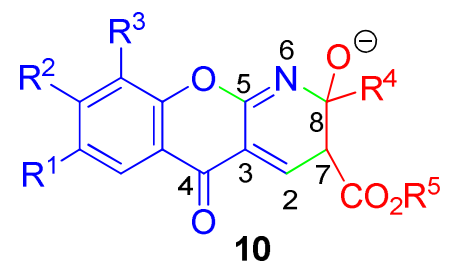

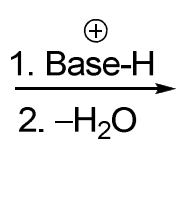<smiles>[R]c1cc2c(=O)c3cc(C(=O)O[Na])c([R])nc3oc2c([R])c1[R]</smiles>

Scheme 1. Mechanistic rationalization for the formation of chromenopyridines $\mathbf{3 / 5}$. The numbering used is arbitrary for the understanding the skeletal carbon rearrangement.

\section{Structure assignment of the new compounds}

The assigned molecular structures of all chromenopyridines $\mathbf{3}$ and $\mathbf{5}$ were based on rigorous spectroscopic analysis including IR, MS, NMR $\left({ }^{1} \mathrm{H},{ }^{13} \mathrm{C}, \mathrm{H}-\mathrm{H}\right.$ COSY, HMQC and HMBC) and elemental analysis data.

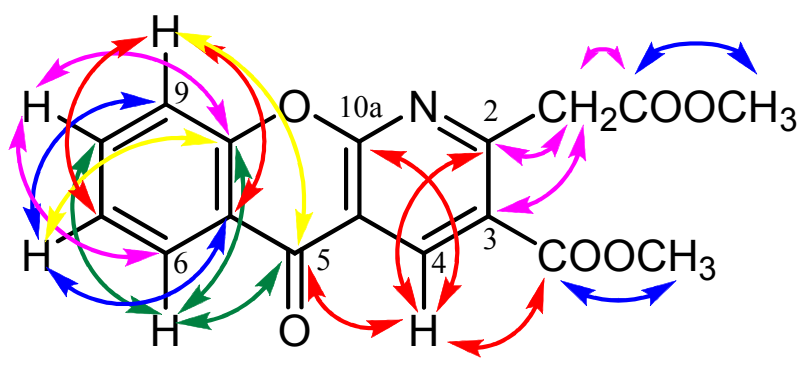

Figure 1. HMBC correlations via ${ }^{2} J_{C H},{ }^{3} J_{C H}$ and ${ }^{4} J_{C H}$ (in yellow color) couplings observed in compound 3a, and the numbering of its skeleton.

A typical ${ }^{1} \mathrm{H}$ NMR spectrum of the chromenopyridine derivative 3a contains a characteristic sequence of four aromatic chromone protons, at $\delta 7.63$ (dd), 7.82 (ddd), 7.49 (ddd) and 8.33 (dd) with the corresponding carbons at 118.6, 136.1, 125.3, and $126.9 \mathrm{ppm}$, respectively. Very characteristic and of good diagnostic value is the one-proton signal at $\delta 9.32$ corresponding to the 4-H with its carbon at $141.1 \mathrm{ppm}$ correlating in $\mathrm{HMBC}$ with carbons at 160.6 (C10a), $161.6(\mathrm{C} 2)$, 
165.0 (3-CO), and 176.7 (C5) ppm (Figure 1). The ester methyl protons correlate with the ester carbonyl carbons and the 2- $\mathrm{CH}_{2}$ protons with carbons at $161.6(\mathrm{C} 2), 123.4(\mathrm{C} 3)$, and at 170.1 (2$\mathrm{CO}$ ) distinguishing thus their neighbor carbonyl group. In the case of structure $\mathbf{1 1}$ (Figure 2) the singlet at $\delta 9.32$ corresponding to the proton 4-H could not have HMBC correlation with 1-CO carbonyl carbon, due to the five intervening bonds, proving thus that the structure of 3a corresponds beyond doubt to the isolated products.<smiles>[R]Cc1c([R])ncc2c(=O)c3cc([R])c([R])c([Y])c3oc12</smiles>

Figure 2. Alternative product not isolated.

\section{Biological evaluation}

The synthesized chromenopyridines $\mathbf{3}$ and $\mathbf{5}$ contain two pharmacophore groups: i) the chromone ring and ii) the pyridine ring. Thus, they might be able to behave as hybrid molecules in terms of biological activity. Therefore, some of the new compounds were screened for their antioxidant activity according to a previously reported methodology, ${ }^{23,24}$ and more specifically, for their reducing ability (RA) by interaction with the stable free radical 1,1-diphenylpicrylhydrazyl (DPPH) and for their inhibition on lipid peroxidation by interaction with the water soluble azo compound 2,2"-azobis(2-amidinopropane) dihydrochloride (AAPH) and the results are compared to well known antioxidant agents e.g. nordihydroguairetic acid (NDGA), and 6-hydroxy-2,5,7,8tetramethylchroman-2-carboxylic acid (trolox). Also, since both pharmacophores have been separately implicated in the inhibition of lipoxygenase a preliminary test in vitro for their anti-LOX activity should be of interest. ${ }^{18,24}$ Each in vitro experiment was performed at least in triplicate, the results were averaged and the standard deviation of absorbance was less than $10 \%$ of the mean. The results are presented in Table 4.

The tested compounds present limited reducing activity expressed as RA\%, through their interaction with DPPH. However, using AAPH as azo initiator of lipid peroxidation, all tested compounds (3a, 3c, 3d, 3f, 3g, and 5f) except 5c were found to highly inhibit lipid peroxidation. Concerning the inhibition of soybean lipoxygenase activity compounds $\mathbf{3 c}$, and $\mathbf{3 g}$ seem to be the most biologically active and their anti-lipid peroxidation activity proceeds in parallel to their enzyme inhibition. Therefore, these two derivatives could be used as lead for the design of new potent antioxidants. 
Table 4. Reducing activity (RA\%) by interaction with DPPH; In vitro inhibition values $\left(\mathrm{IC}_{50}\right)$ of lipid peroxidation induced by AAPH; In vitro \% inhibition of soybean lipoxygenase (LOX \%)

\begin{tabular}{cccccc}
\hline Product & \multicolumn{2}{c}{$\begin{array}{c}\text { DPPH }(\mathrm{RA} \%) \\
\text { at } 100 \mu \mathrm{M} \\
20 \mathrm{~min}\end{array}$} & $\begin{array}{c}\text { AAPH } \\
\mathrm{IC}_{50} \mu \mathrm{M}\end{array}$ & \multicolumn{2}{c}{ LOX } \\
& & & & $\begin{array}{c}\text { Inh \% } \\
100 \mu \mathrm{M}\end{array}$ & $\mathrm{IC}_{50} \mu \mathrm{M}$ \\
\hline $\mathbf{3 a}$ & 14 & 9 & 85 & 43 & \\
$\mathbf{3 c}$ & 14 & 12 & 76 & 61 & 82 \\
$\mathbf{3 d}$ & 15 & 12 & 73 & 35 & \\
$\mathbf{3 f}$ & 16 & 14 & 75 & 30 & \\
$\mathbf{3 g}$ & 12 & 12 & 75 & 59 & 85 \\
$\mathbf{5 c}$ & 2 & 9 & $19 \%$ at & no & \\
& & & $100 \mu \mathrm{M}$ & & \\
$\mathbf{5 f}$ & 8 & no & 80 & 17 & \\
NDGA & 83 & 86 & & 93 & \\
Trolox & & & 55 & & \\
\hline
\end{tabular}

\section{Conclusions}

In the present study an investigation concerning the optimization of the reaction conditions under normal heating and, alternatively, under sonication leading to the synthesis of chromeno[2,3b]pyridines $\mathbf{3}$ and $\mathbf{5}$ is described. The biological results showed that all tested compounds except $\mathbf{5 c}$ were found to highly inhibit lipid peroxidation. Compounds $\mathbf{3 c}$ and $\mathbf{3 g}$ are promising leads chromenopyridines combining increased anti-lipid peroxidation and lipoxygenase inhibitory activities. Full assignment of all ${ }^{1} \mathrm{H}$ and ${ }^{13} \mathrm{C}$ NMR chemical shifts has been unambiguously achieved. The earlier proposed plausible mechanistic scheme was confirmed.

\section{Experimental Section}

General. Melting points were measured on a Kofler hot-stage and are uncorrected. Column chromatography was carried out using Merck silica gel (70-230 mesh). TLC was performed using precoated silica gel glass plates $0.25 \mathrm{~mm}$ containing fluorescent indicator $\mathrm{UV}_{254}$ purchased from Macherey-Nagel using a 3:1 mixture of petroleum ether-ethyl acetate. Petroleum ether refers to the fraction boiling between 60 and $80{ }^{\circ} \mathrm{C}$. NMR spectra were recorded at $25^{\circ} \mathrm{C}$ on a Bruker AVANCE III 300 spectrometer at $300 \mathrm{MHz}$ for ${ }^{1} \mathrm{H}$ and $75 \mathrm{MHz}$ for ${ }^{13} \mathrm{C}$, using $\mathrm{CDCl}_{3}$, DMSO- $d_{6}$ or their mixture as solvent, as indicated. Some samples are measured on a Varian $500 \mathrm{MHz}$ spectrometer, as indicated. The chemical shifts are expressed in $\delta$ values (ppm) relative to TMS as internal standard 
for ${ }^{1} \mathrm{H}$ and relative to TMS (0.00 ppm), or to $\mathrm{CDCl}_{3}$ (77.05 ppm) or to DMSO- $d_{6}(40.0 \mathrm{ppm})$ for ${ }^{13} \mathrm{C}$ NMR spectra. Coupling constants ${ }^{n} J$ are reported in Hz. IR spectra were recorded on a Perkin-Elmer 1600 series FTIR spectrometer and are reported in wave numbers $\left(\mathrm{cm}^{-1}\right)$. Low resolution LC-MS (ESI, $1.65 \mathrm{eV}$ ) mass spectra were recorded on LC-MS-2010 EV system (Shimadzu). Elemental analyses performed with a Perkin-Elmer 2400-II CHN analyzer. Structural assignments of the derived compounds were established by analysis of their IR, MS and NMR spectra $\left({ }^{1} \mathrm{H},{ }^{13} \mathrm{C}\right.$, COSY $\mathrm{H}-\mathrm{H}, \mathrm{HMQC}$ and HMBC). Ultrasound for sonication is generated using the instrument: ELMA TRANSSONIC T310 (Germany); operating frequency $35 \mathrm{kHz}, 220 \mathrm{~V}, 50 \mathrm{~Hz}, 140 \mathrm{~mA}$; bath dimensions $190 \times 85 \times 55 \mathrm{~mm}$.

5-Ox0-5H-chromeno[2,3-b]pyridine-carboxylates. A solution of acetonedicarboxylate $(0.5 \mathrm{mmol})$ and DABCO $(0.5 \mathrm{mmol})$ in ethanol $(5 \mathrm{~mL})$ were refluxed for $15 \mathrm{~min}$, whereupon 3-cyanochromone $(0.5 \mathrm{mmol})$ was added and the reaction mixture was refluxed further for $4 \mathrm{~h}$. Upon cooling the product was crystallized, filtered by Gooch funnel, washed on the funnel with diethyl ether and recrystallized from ethanol. Alternatively, the reaction mixture in a small amount of ethanol $(2 \mathrm{~mL})$ was subjected to ultrasound irradiation for 10 min using the water bath at $20{ }^{\circ} \mathrm{C}$, whereupon after initial solvation of the reactants the chromenopyridine product was crystallized out.

Methyl 2-(2-methoxy-2-oxoethyl)-5-oxo-5H-chromeno[2,3-b]pyridine-3-carboxylate (3a). Yield: $0.139 \mathrm{~g}(85 \%)$, yellow solid, mp $170-172{ }^{\circ} \mathrm{C}$ (ethanol); IR $\left(\mathrm{KBr}, v_{\max }, \mathrm{cm}^{-1}\right): 1731(\mathrm{C}=\mathrm{O})$, $1676(\mathrm{C}=\mathrm{O}) .{ }^{1} \mathrm{H}$ NMR $\left(300 \mathrm{MHz}, \mathrm{CDCl}_{3}\right): \delta 3.75\left(\mathrm{~s}, 3 \mathrm{H}, 2-\mathrm{OCH}_{3}\right), 3.97$ (s, 3H, 3- $\left.\mathrm{OCH}_{3}\right), 4.42(\mathrm{~s}$, 2H, 2- $\mathrm{CH}_{2}$ ), 7.49 (ddd, $J$ 8.0, 7.4, $\left.0.5 \mathrm{~Hz}, 1 \mathrm{H}, 7-\mathrm{H}\right), 7.63$ (dd, $J 8.0,0.5 \mathrm{~Hz}, 1 \mathrm{H}, 9-\mathrm{H}$ ), 7.82 (ddd, $J$ 8.0, 7.4, $1.2 \mathrm{~Hz}, 1 \mathrm{H}, 8-\mathrm{H}), 8.33$ (dd, J 8.0, $1.2 \mathrm{~Hz}, 1 \mathrm{H}, 6-\mathrm{H}), 9.32$ (s, 1H, 4-H). ${ }^{13} \mathrm{C} \mathrm{NMR}(75 \mathrm{MHz}$, $\left.\mathrm{CDCl}_{3}\right): \delta 43.8\left(2-\mathrm{CH}_{2}\right), 52.3\left(2-\mathrm{OCH}_{3}\right), 52.7\left(3-\mathrm{OCH}_{3}\right), 115.2(\mathrm{C} 4 \mathrm{a}), 118.6(\mathrm{C} 9), 121.6(\mathrm{C} 5 \mathrm{a})$, 123.4 (C3), 125.3 (C7), 126.9 (C6), 136.1 (C8), 141.1 (C4), 155.5 (C9a), 160.6 (C10a), 161.6 (C2), 165.0 (3-CO), 170.1 (2-CO), 176.7 (C5). LC-MS (ESI, $1.65 \mathrm{eV}): \mathrm{m} / z(\%) 382[98,(\mathrm{M}+\mathrm{Na}+$ $\left.\mathrm{MeOH})^{+}\right], 366\left[70(\mathrm{M}+\mathrm{K})^{+}\right], 350\left[100,(\mathrm{M}+\mathrm{Na})^{+}\right], 328\left[10,(\mathrm{M}+\mathrm{H})^{+}\right]$. Anal. Calcd for $\mathrm{C}_{17} \mathrm{H}_{13} \mathrm{NO}_{6}$ (327.29): C, 62.39; H, 4.00; N, 4.28. Found: C, 62.34; H, 4.12; N, 4.34.

Ethyl 2-(2-ethoxy-2-oxoethyl)-5-oxo-5H-chromeno[2,3-b]pyridine-3-carboxylate (3b).Yield: $0.146 \mathrm{~g}(82 \%)$, yellow solid, $\mathrm{mp} 153-155^{\circ} \mathrm{C}$ (ethanol); IR $\left(\mathrm{KBr}, v_{\max }, \mathrm{cm}^{-1}\right): 1724(\mathrm{C}=\mathrm{O}), 1673$ $(\mathrm{C}=\mathrm{O}) .{ }^{1} \mathrm{H}$ NMR $\left(300 \mathrm{MHz}, \mathrm{CDCl}_{3}\right): \delta 1.26\left(\mathrm{t}, J 7.1 \mathrm{~Hz}, 3 \mathrm{H}, 2-\mathrm{OCH}_{2} \mathrm{CH}_{3}\right), 1.44(\mathrm{t}, J 7.1 \mathrm{~Hz}, 3 \mathrm{H}, 3-$ $\left.\mathrm{OCH}_{2} \mathrm{CH}_{3}\right), 4.20\left(\mathrm{q}, J 7.1 \mathrm{~Hz}, 2 \mathrm{H}, 2-\mathrm{OCH}_{2} \mathrm{CH}_{3}\right), 4.41\left(\mathrm{~s}, 2 \mathrm{H}, 2-\mathrm{CH}_{2}\right), 4.42(\mathrm{q}, J 7.1 \mathrm{~Hz}, 2 \mathrm{H}, 3-$ $\mathrm{OCH}_{2} \mathrm{CH}_{3}$ ), 7.48 (ddd, $J$ 8.0, 7.3, $\left.0.7 \mathrm{~Hz}, 1 \mathrm{H}, 7-\mathrm{H}\right), 7.63(\mathrm{dd}, J$ 8.3, $0.7 \mathrm{~Hz}, 1 \mathrm{H}, 9-\mathrm{H}), 7.82$ (ddd, $J$ 8.3, 7.3, $1.6 \mathrm{~Hz}, 1 \mathrm{H}, 8-\mathrm{H}), 8.34$ (dd, J 8.0, $1.6 \mathrm{~Hz}, 1 \mathrm{H}, 6-\mathrm{H}), 9.31$ (s, $1 \mathrm{H}, 4-\mathrm{H}) .{ }^{13} \mathrm{C} \mathrm{NMR}(75 \mathrm{MHz}$, $\left.\mathrm{CDCl}_{3}\right): \delta 14.16\left(2-\mathrm{CH}_{2} \mathrm{CH}_{3}\right), 14.24\left(3-\mathrm{OCH}_{2} \mathrm{CH}_{3}\right), 44.1\left(2-\mathrm{CH}_{2}\right), 61.2\left(2-\mathrm{OCH}_{2} \mathrm{CH}_{3}\right), 61.9$ (3$\mathrm{OCH}_{2} \mathrm{CH}_{3}$ ), 115.1 (C4a), 118.6 (C9), 121.7 (C5a), 123.9 (C3), 125.3 (C7), 126.9 (C6), 136.0 (C8), 140.9 (C4), 155.6 (C9a), 160.5 (C10a), 161.7 (C2), 164.6 (3-CO), 169.6 (2-CO), 176.8 (C5). LCMS (ESI, $1.65 \mathrm{eV}): \mathrm{m} / \mathrm{z}(\%) 733\left[60,(2 \times \mathrm{M}+\mathrm{Na})^{+}\right], 410\left[100,(\mathrm{M}+\mathrm{Na}+\mathrm{MeOH})^{+}\right], 378[95,(\mathrm{M}+$ $\left.\mathrm{Na})^{+}\right], 356\left[20,(\mathrm{M}+\mathrm{H})^{+}\right]$. Anal. Calcd for $\mathrm{C}_{19} \mathrm{H}_{17} \mathrm{NO}_{6}$ (355.35): C, 64.22; H, 4.82; N, 3.94. Found: C, 64.44; H, 4.70; N, 4.05.

Methyl 2-(2-methoxy-2-oxoethyl-7-methyl)-5-oxo-5H-chromeno[2,3-b]pyridine-3-carboxylate (3c). Yield: $0.136 \mathrm{~g}(80 \%)$, yellow solid, $\mathrm{mp} 150-151{ }^{\circ} \mathrm{C}$ (ethanol); IR $\left(\mathrm{KBr}, v_{\max }, \mathrm{cm}^{-1}\right): 1739(\mathrm{C}=\mathrm{O})$, 
$1680(\mathrm{C}=\mathrm{O}), 1670(\mathrm{C}=\mathrm{O}) .{ }^{1} \mathrm{H}$ NMR $\left(300 \mathrm{MHz}, \mathrm{CDCl}_{3}\right): \delta 2.49\left(\mathrm{~s}, 3 \mathrm{H}, 7-\mathrm{CH}_{3}\right), 3.74(\mathrm{~s}, 3 \mathrm{H}, 2-$ $\left.\mathrm{OCH}_{3}\right), 3.96\left(\mathrm{~s}, 3 \mathrm{H}, 3-\mathrm{OCH}_{3}\right), 4.41\left(\mathrm{~s}, 2 \mathrm{H}, 2-\mathrm{CH}_{2}\right), 7.51(\mathrm{~d}, J 8.6 \mathrm{~Hz}, 1 \mathrm{H}, 9-\mathrm{H}), 7.61$ (dd, J 8.6, 1.9 $\mathrm{Hz}, 1 \mathrm{H}, 8-\mathrm{H}), 8.10$ (d, J $1.9 \mathrm{~Hz}, 1 \mathrm{H}, 6-\mathrm{H}), 9.30$ (s, 1H, 4-H). ${ }^{13} \mathrm{C} \mathrm{NMR}\left(75 \mathrm{MHz}, \mathrm{CDCl}_{3}\right): \delta 20.9(7-$ $\left.\mathrm{CH}_{3}\right), 43.8\left(2-\mathrm{CH}_{2}\right), 52.3\left(2-\mathrm{OCH}_{3}\right), 52.7\left(3-\mathrm{OCH}_{3}\right), 115.2(\mathrm{C} 4 \mathrm{a}), 118.4$ (C9), $121.4(\mathrm{C} 5 \mathrm{a}), 123.3$ (C3), 126.3 (C6), 135.4 (C7), 137.3 (C8), 141.1 (C4), 153.9 (C9a), 160.7 (C10a), 161.5 (C2), 165.1 (3-CO), 170.0 (2-CO), 176.8 (C-5). LC-MS (ESI, $1.65 \mathrm{eV}): \mathrm{m} / \mathrm{z}(\%) 412$ [80, $\left.(\mathrm{M}+\mathrm{K}+\mathrm{MeOH})^{+}\right]$, $396\left[78,(\mathrm{M}+\mathrm{Na}+\mathrm{MeOH})^{+}\right], 380\left[31,(\mathrm{M}+\mathrm{K})^{+}\right], 364\left[100,(\mathrm{M}+\mathrm{Na})^{+}\right], 342\left[18,(\mathrm{M}+\mathrm{H})^{+}\right]$. Anal. Calcd for $\mathrm{C}_{18} \mathrm{H}_{15} \mathrm{NO}_{6}$ (341.31): C, 63.34; H, 4.43; N, 4.10. Found: C, 63.22; H, 4.51; N, 4.18.

Ethyl 2-(2-ethoxy-2-oxoethyl-7-methyl)-5-oxo-5H-chromeno[2,3-b]pyridine-3-carboxylate (3d).Yield: $0.149 \mathrm{~g}(81 \%)$, yellow solid, $\mathrm{mp} 131-133{ }^{\circ} \mathrm{C}$ (ethanol); IR $\left(\mathrm{KBr}, v_{\max }, \mathrm{cm}^{-1}\right): 1731$ $(\mathrm{C}=\mathrm{O}), 1670(\mathrm{C}=\mathrm{O}) .{ }^{1} \mathrm{H} \mathrm{NMR}\left(300 \mathrm{MHz}, \mathrm{CDCl}_{3}\right): \delta 1.26\left(\mathrm{t}, J 7.1 \mathrm{~Hz}, 3 \mathrm{H}, 2-\mathrm{CH}_{2} \mathrm{CH}_{3}\right), 1.43(\mathrm{t}, J 7.1$ $\left.\mathrm{Hz}, 3 \mathrm{H}, 3-\mathrm{CH}_{2} \mathrm{CH}_{3}\right), 2.50\left(\mathrm{~s}, 3 \mathrm{H}, 7-\mathrm{CH}_{3}\right), 4.19$ (q, J 7.1 Hz, 2H, 2- $\left.\mathrm{CH}_{2} \mathrm{CH}_{3}\right), 4.40\left(\mathrm{~s}, 2 \mathrm{H}, 2-\mathrm{CH}_{2}\right)$, $4.41\left(\mathrm{q}, J 7.1 \mathrm{~Hz}, 2 \mathrm{H}, 3-\mathrm{CH}_{2} \mathrm{CH}_{3}\right), 7.51(\mathrm{~d}, J 8.5 \mathrm{~Hz}, 1 \mathrm{H}, 9-\mathrm{H}), 7.61(\mathrm{dd}, J 8.5,1.9 \mathrm{~Hz}, 1 \mathrm{H}, 8-\mathrm{H})$, $8.10(\mathrm{~d}, J 1.9 \mathrm{~Hz}, 1 \mathrm{H}, 6-\mathrm{H}), 9.29(\mathrm{~s}, 1 \mathrm{H}, 4-\mathrm{H}) .{ }^{13} \mathrm{C} \mathrm{NMR}\left(75 \mathrm{MHz}, \mathrm{CDCl}_{3}\right): \delta 14.17\left(2-\mathrm{CH}_{2} \mathrm{CH}_{3}\right)$, $14.26\left(3-\mathrm{OCH}_{2} \mathrm{CH}_{3}\right), 20.9\left(7-\mathrm{CH}_{3}\right), 44.1\left(2-\mathrm{CH}_{2}\right), 61.2\left(2-\mathrm{OCH}_{2} \mathrm{CH}_{3}\right), 61.9\left(3-\mathrm{CH}_{2} \mathrm{CH}_{3}\right), 115.1$ (C4a), 118.4 (C9), 121.4 (C5a), 123.8 (C3), 126.3 (C6), 135.3 (C7), 137.2 (C8), 140.9 (C4), 153.9 (C9a), 160.6 (C10a), 161.6 (C2), 164.7 (3-CO), 169.6 (2-CO), 176.9 (C5). LC-MS (ESI, 1.65 eV): $m / z(\%) 424\left[65,(\mathrm{M}+\mathrm{Na}+\mathrm{MeOH})^{+}\right], 392\left[100,(\mathrm{M}+\mathrm{Na})^{+}\right]$. Anal. Calcd for $\mathrm{C}_{20} \mathrm{H}_{19} \mathrm{NO}_{6}(369.37)$ : C, 65.03; H, 5.18; N, 3.79. Found: C, 65.22; H, 5.11; N, 3.88 .

Methyl 2-(2-methoxy-2-oxoethyl-7,8-dimethyl)-5-oxo-5H-chromeno[2,3-b]pyridine-3-carboxylate (3e). Yield: $0.153 \mathrm{~g}(86 \%)$, yellow solid, $\mathrm{mp} 175-177{ }^{\circ} \mathrm{C}$ (ethanol); IR $\left(\mathrm{KBr}, v_{\max }, \mathrm{cm}^{-1}\right)$ : 1735 $(\mathrm{C}=\mathrm{O}), 1725(\mathrm{C}=\mathrm{O}), 1673(\mathrm{C}=\mathrm{O}) .{ }^{1} \mathrm{H} \mathrm{NMR}\left(300 \mathrm{MHz}, \mathrm{CDCl}_{3}\right): \delta 2.39\left(\mathrm{~s}, 3 \mathrm{H}, 8-\mathrm{CH}_{3}\right), 2.44(\mathrm{~s}, 3 \mathrm{H}$, 7- $\left.\mathrm{CH}_{3}\right), 3.74\left(\mathrm{~s}, 3 \mathrm{H}, 2-\mathrm{OCH}_{3}\right), 3.96\left(\mathrm{~s}, 3 \mathrm{H}, 3-\mathrm{OCH}_{3}\right), 4.41\left(\mathrm{~s}, 2 \mathrm{H}, 2-\mathrm{CH}_{2}\right), 7.37(\mathrm{~s}, 1 \mathrm{H}, 9-\mathrm{H}), 8.04(\mathrm{~s}$, $1 \mathrm{H}, 6-\mathrm{H}), 9.30(\mathrm{~s}, 1 \mathrm{H}, 4-\mathrm{H}) .{ }^{13} \mathrm{C} \mathrm{NMR}\left(75 \mathrm{MHz}, \mathrm{CDCl}_{3}\right): \delta 19.3\left(8-\mathrm{CH}_{3}\right), 20.8\left(7-\mathrm{CH}_{3}\right), 43.8(2-$ $\left.\mathrm{CH}_{2}\right), 52.3\left(2-\mathrm{OCH}_{3}\right), 52.7\left(3-\mathrm{OCH}_{3}\right), 115.3(\mathrm{C} 4 \mathrm{a}), 118.7(\mathrm{C} 9), 119.4(\mathrm{C} 5 \mathrm{a}), 123.1(\mathrm{C} 3), 126.5$ (C6), 134.7 (C7), 141.0 (C4), 147.1 (C8), 154.0 (C9a), 160.7 (C10a), 161.2 (C2), 165.1 (3-CO), 170.1 (2-CO), 176.6 (C-5). LC-MS (ESI, $1.65 \mathrm{eV}): \mathrm{m} / z(\%) 733\left[100,(2 \times \mathrm{M}+\mathrm{N \alpha})^{+}\right], 410[60,(\mathrm{M}+$ $\left.\mathrm{Na}+\mathrm{MeOH})^{+}\right], 394\left[31,(\mathrm{M}+\mathrm{K})^{+}\right], 378\left[65,(\mathrm{M}+\mathrm{Na})^{+}\right], 356\left[20,(\mathrm{M}+\mathrm{H})^{+}\right]$. Anal. Calcd for $\mathrm{C}_{19} \mathrm{H}_{17} \mathrm{NO}_{6}$ (355.34): C, 64.22; H, 4.82; N, 3.94. Found: C, 63.22; H, 4.51; N, 4.18.

Methyl 2-(2-methoxy-2-oxoethyl-7-chloro)-5-oxo-5H-chromeno[2,3-b]pyridine-3-carboxylate (3f). Yield: $0.141 \mathrm{~g}(78 \%)$, yellow solid, mp 188-190 ${ }^{\circ} \mathrm{C}$ (ethanol); $\mathrm{IR}\left(\mathrm{KBr}, v_{\max }, \mathrm{cm}^{-1}\right): 1732$ $(\mathrm{C}=\mathrm{O}), 1721(\mathrm{C}=\mathrm{O}), 1673(\mathrm{C}=\mathrm{O}) .{ }^{1} \mathrm{H}$ NMR $\left(300 \mathrm{MHz}, \mathrm{CDCl}_{3}\right): \delta 3.74\left(\mathrm{~s}, 3 \mathrm{H}, 2-\mathrm{OCH}_{3}\right), 3.97(\mathrm{~s}$, $\left.3 \mathrm{H}, 3-\mathrm{OCH}_{3}\right), 4.42\left(\mathrm{~s}, 2 \mathrm{H}, 2-\mathrm{CH}_{2}\right), 7.58(\mathrm{~d}, J 8.9 \mathrm{~Hz}, 1 \mathrm{H}, 9-\mathrm{H}), 7.74$ (dd, J 8.9, $\left.2.5 \mathrm{~Hz}, 1 \mathrm{H}, 8-\mathrm{H}\right)$, $8.28(\mathrm{~d}, J 2.5 \mathrm{~Hz}, 1 \mathrm{H}, 6-\mathrm{H}), 9.29(\mathrm{~s}, 1 \mathrm{H}, 4-\mathrm{H}) .{ }^{13} \mathrm{C} \mathrm{NMR}\left(75 \mathrm{MHz}, \mathrm{CDCl}_{3}\right): \delta 43.6\left(2-\mathrm{CH}_{2}\right), 52.2(2-$ $\left.\mathrm{OCH}_{3}\right), 52.6\left(3-\mathrm{OCH}_{3}\right), 114.7(\mathrm{C} 4 \mathrm{a}), 120.3(\mathrm{C} 9), 122.5(\mathrm{C} 5 \mathrm{a}), 123.7(\mathrm{C} 3), 126.1(\mathrm{C} 6), 131.2(\mathrm{C} 7)$, 136.0 (C8), 141.0 (C4), 153.8 (C9a), 160.3 (C10a), 161.8 (C2), 164.7 (3-CO), 169.7 (2-CO), 175.6 (C-5). LC-MS (ESI, $1.65 \mathrm{eV}): \mathrm{m} / z(\%)$ 416/418 [100, $\left.(\mathrm{M}+\mathrm{Na}+\mathrm{MeOH})^{+}\right], 400 / 402[28,(\mathrm{M}+$ $\mathrm{K})^{+}$], 384/386 [75, $\left.(\mathrm{M}+\mathrm{Na})^{+}\right], 362 / 364\left[5,(\mathrm{M}+\mathrm{H})^{+}\right.$]. Anal. Calcd for $\mathrm{C}_{17} \mathrm{H}_{12} \mathrm{ClNO}_{6}$ (361.73): $\mathrm{C}$, 56.45; H, 3.34; N, 3.87. Found: C, 56.52; H, 3.41; N, 3.73.

Ethyl 2-(2-ethoxy-2-oxoethyl-7-chloro)-5-oxo-5H-chromeno[2,3-b]pyridine-3-carboxylate (3g). Yield: $0.168 \mathrm{~g}(86 \%)$, yellow solid, mp $144-146{ }^{\circ} \mathrm{C}$ (ethanol); IR $\left(\mathrm{KBr}, v_{\max }, \mathrm{cm}^{-1}\right): 1728(\mathrm{C}=\mathrm{O})$, 
$1667(\mathrm{C}=\mathrm{O}) .{ }^{1} \mathrm{H}$ NMR $\left(300 \mathrm{MHz}, \mathrm{CDCl}_{3}\right): \delta 1.26\left(\mathrm{t}, J 7.1 \mathrm{~Hz}, 3 \mathrm{H}, 2-\mathrm{CH}_{2} \mathrm{CH}_{3}\right), 1.44(\mathrm{t}, J 7.1 \mathrm{~Hz}$, $\left.3 \mathrm{H}, 3-\mathrm{CH}_{2} \mathrm{CH}_{3}\right), 4.19\left(\mathrm{q}, J 7.1 \mathrm{~Hz}, 2 \mathrm{H}, 2-\mathrm{CH}_{2} \mathrm{CH}_{3}\right), 4.40\left(\mathrm{~s}, 2 \mathrm{H}, 2-\mathrm{CH}_{2}\right), 4.42(\mathrm{q}, J 7.1 \mathrm{~Hz}, 2 \mathrm{H}, 3-$ $\left.\mathrm{CH}_{2} \mathrm{CH}_{3}\right), 7.59(\mathrm{~d}, J 8.9 \mathrm{~Hz}, 1 \mathrm{H}, 9-\mathrm{H}), 7.76(\mathrm{dd}, J 8.9,2.5 \mathrm{~Hz}, 1 \mathrm{H}, 8-\mathrm{H}), 8.27(\mathrm{~d}, J 2.5 \mathrm{~Hz}, 1 \mathrm{H}, 6-\mathrm{H})$, 9.26 (s, $1 \mathrm{H}, 4-\mathrm{H}) .{ }^{13} \mathrm{C} \mathrm{NMR}\left(75 \mathrm{MHz}, \mathrm{CDCl}_{3}\right): \delta 14.0\left(2-\mathrm{CH}_{2} \mathrm{CH}_{3}\right), 14.1\left(3-\mathrm{CH}_{2} \mathrm{CH}_{3}\right), 43.9\left(2-\mathrm{CH}_{2}\right)$, $61.1\left(2-\mathrm{CH}_{2} \mathrm{CH}_{3}\right), 61.9\left(3-\mathrm{CH}_{2} \mathrm{CH}_{3}\right), 114.6$ (C4a), 120.3 (C9), 124.1 (C3), 125.8 (C5a), 127.5 (C6), 130.9 (C7), 136.0 (C8), 140.7 (C4), 155.0 (C9a), 160.1 (C10a), 161.8 (C2), 164.2 (3-CO), 169.3 (2CO), 175.6 (C5). LC-MS (ESI, $1.65 \mathrm{eV}): \mathrm{m} / z(\%)$ 444/446 [55, (M + Na + MeOH $\left.)^{+}\right], 428 / 430[15$, $\left.(\mathrm{M}+\mathrm{K})^{+}\right], 412 / 414\left[50,(\mathrm{M}+\mathrm{Na})^{+}\right], 390 / 392\left[5,(\mathrm{M}+\mathrm{H})^{+}\right], 313 / 315\left[100,(\mathrm{M}-76)^{+}\right]$. Anal. Calcd for $\mathrm{C}_{19} \mathrm{H}_{16} \mathrm{ClNO}_{6}$ (389.79): C, 58.55; H, 4.14; N, 3.59. Found: C, 58.62; H, 4.32; N, 3.68.

Methyl 2-(2-methoxy-2-oxoethyl-7,9-dichloro)-5-oxo-5H-chromeno[2,3-b]pyridine-3-carboxylate (3h). Yield: $0.157 \mathrm{~g}$ (79\%), yellow solid, $\mathrm{mp} 166-168{ }^{\circ} \mathrm{C}$ (ethanol); IR $\left(\mathrm{KBr}, v_{\max }, \mathrm{cm}^{-1}\right)$ : 1725 $(\mathrm{C}=\mathrm{O}), 1677(\mathrm{C}=\mathrm{O}) .{ }^{1} \mathrm{H}$ NMR $\left(300 \mathrm{MHz}, \mathrm{CDCl}_{3}\right): \delta 3.73\left(\mathrm{~s}, 3 \mathrm{H}, 2-\mathrm{OCH}_{3}\right), 3.97\left(\mathrm{~s}, 3 \mathrm{H}, 3-\mathrm{OCH}_{3}\right)$, $4.45\left(\mathrm{~s}, 2 \mathrm{H}, 2-\mathrm{CH}_{2}\right), 7.85(\mathrm{~d}, J 2.3 \mathrm{~Hz}, 1 \mathrm{H}, 8-\mathrm{H}), 8.20(\mathrm{~d}, J 2.3 \mathrm{~Hz}, 1 \mathrm{H}, 6-\mathrm{H}), 9.28(\mathrm{~s}, 1 \mathrm{H}, 4-\mathrm{H}) .{ }^{13} \mathrm{C}$ NMR $\left(75 \mathrm{MHz}, \mathrm{CDCl}_{3}+\mathrm{DMSO}-d_{6}\right): \delta 43.4\left(2-\mathrm{CH}_{2}\right), 51.9\left(2-\mathrm{OCH}_{3}\right), 52.6\left(3-\mathrm{OCH}_{3}\right), 114.3(\mathrm{C} 4 \mathrm{a})$, 123.0 (C5a), 123.2 (C3), 124.1 (C9), 124.4 (C6), 130.4 (C7), 135.5 (C8), 140.5 (C4), 149.7 (C9a), 159.6 (C-10a), 161.9 (C2), 164.3 (C2”), 169.4 (C2'), 174.7 (C-5). LC-MS (ESI, 1.65 eV): m/z (\%) 450/452/454 [60, $\left.(\mathrm{M}+\mathrm{Na}+\mathrm{MeOH})^{+}\right], 434 / 436 / 438\left[50,(\mathrm{M}+\mathrm{K})^{+}\right], 418 / 420 / 422\left[100,(\mathrm{M}+\mathrm{Na})^{+}\right]$, 396/398/400 [10, $\left.(\mathrm{M}+\mathrm{H})^{+}\right]$. Anal. Calcd for $\mathrm{C}_{17} \mathrm{H}_{11} \mathrm{Cl}_{2} \mathrm{NO}_{6}$ (396.18): C, 51.54; H, 2.80; N, 3.54 . Found: C, 51.62; H, 2.73; N, 3.58.

Ethyl 2-methyl-5-oxo-5H-chromeno[2,3-b]pyridine-3-carboxylate (5a). Yield: $0.108 \mathrm{~g}$ (76\%), yellow solid, mp 161-163 ${ }^{\circ} \mathrm{C}$ (ethanol); IR $\left(\mathrm{KBr}, v_{\max }, \mathrm{cm}^{-1}\right)$ : $1731(\mathrm{C}=\mathrm{O}), 1676(\mathrm{C}=\mathrm{O}) .{ }^{1} \mathrm{H}$ NMR $\left(300 \mathrm{MHz}, \mathrm{CDCl}_{3}\right): \delta 1.45\left(\mathrm{t}, J 7.2 \mathrm{~Hz}, 3 \mathrm{H}, 3-\mathrm{CH}_{2} \mathrm{CH}_{3}\right), 2.99\left(\mathrm{~s}, 3 \mathrm{H}, 2-\mathrm{CH}_{3}\right), 4.44(\mathrm{q}, J 7.2 \mathrm{~Hz}, 2 \mathrm{H}$, 3- $\mathrm{CH}_{2} \mathrm{CH}_{3}$ ), 7.47 (ddd, $J$ 8.0, 7.2, $0.8 \mathrm{~Hz}, 1 \mathrm{H}, 7-\mathrm{H}$ ), 7.63 (dd, $J 8.3,0.8 \mathrm{~Hz}, 1 \mathrm{H}, 9-\mathrm{H}$ ), 7.80 (ddd, $J$ 8.3, 7.2, $1.5 \mathrm{~Hz}, 1 \mathrm{H}, 8-\mathrm{H}), 8.32$ (dd, J 8.0, $1.5 \mathrm{~Hz}, 1 \mathrm{H}, 6-\mathrm{H}), 9.20$ (s, 1H, 4-H). ${ }^{13} \mathrm{C} \mathrm{NMR}(75 \mathrm{MHz}$, $\left.\mathrm{CDCl}_{3}\right): \delta 14.1\left(3-\mathrm{CH}_{2} \mathrm{CH}_{3}\right), 25.3\left(2-\mathrm{CH}_{3}\right), 61.6\left(3-\mathrm{CH}_{2} \mathrm{CH}_{3}\right), 114.0(\mathrm{C} 4 \mathrm{a}), 118.6(\mathrm{C} 9), 121.7(\mathrm{C} 5 \mathrm{a})$, 123.5 (C3), 125.0 (C7), 126.6 (C6), 135.6 (C8), 140.5 (C4), 155.5 (C9a), 160.4 (C10a), 161.6 (C2), 164.8 (3-CO), 176.9 (C5). LC-MS (ESI, $1.65 \mathrm{eV}): \mathrm{m} / \mathrm{z}(\%) 338$ [100, (M+ Na + MeOH) $\left.{ }^{+}\right], 306[10$, $\left.(\mathrm{M}+\mathrm{Na})^{+}\right], 284\left[39,(\mathrm{M}+\mathrm{H})^{+}\right]$. Anal. Calcd for $\mathrm{C}_{16} \mathrm{H}_{13} \mathrm{NO}_{4}$ (283.28): C, 67.84; H, 4.63; N, 4.94. Found: C, 67.97; H, 4.75; N, 5.02.

Ethyl 2-phenyl-5-oxo-5H-chromeno[2,3-b]pyridine-3-carboxylate (5b). Yield: $0.121 \mathrm{~g}$ (70\%), yellow solid, mp $146-148{ }^{\circ} \mathrm{C}$ (ethanol); IR $\left(\mathrm{KBr}, v_{\max }, \mathrm{cm}^{-1}\right)$ : $1713(\mathrm{C}=\mathrm{O}), 1664(\mathrm{C}=\mathrm{O}) .{ }^{1} \mathrm{H}$ NMR $\left(500 \mathrm{MHz}, \mathrm{CDCl}_{3}\right): \delta 1.17\left(\mathrm{t}, J 7.2 \mathrm{~Hz}, 3 \mathrm{H}, 3-\mathrm{CH}_{2} \mathrm{CH}_{3}\right), 4.26\left(\mathrm{q}, J 7.2 \mathrm{~Hz}, 2 \mathrm{H}, 3-\mathrm{CH}_{2} \mathrm{CH}_{3}\right), 7.46-$ 7.51 (m, 4H, 3',4',5',7-H), 7.65 (d, J 8.3 Hz, 1H, 9-H), 7.65-7.68 (m, 2H, 2',6'-H), 7.82 (ddd, $J$ 8.3, 7.5, $1.4 \mathrm{~Hz}, 1 \mathrm{H}, \mathrm{H}-8), 8.35$ (dd, $J$ 8.0, $1.4 \mathrm{~Hz}, 1 \mathrm{H}, 6-\mathrm{H}), 9.12$ (s, 1H, 4-H). ${ }^{13} \mathrm{C}$ NMR $(125 \mathrm{MHz}$, $\left.\mathrm{CDCl}_{3}\right): \delta 13.8\left(3-\mathrm{CH}_{2} \mathrm{CH}_{3}\right), 62.0\left(3-\mathrm{CH}_{2} \mathrm{CH}_{3}\right), 114.4(\mathrm{C} 4 \mathrm{a}), 118.6(\mathrm{C} 9), 121.7(\mathrm{C} 5 \mathrm{a}), 125.16(\mathrm{C} 3)$, 125.18 (C7), 126.9 (C6), 128.3 (C2',6'), 128.9 (C3',5'), 130.0 (C4'), 136.0 (C8), 138.4 (C1'), 140.2 (C4), 155.8 (C9a), 160.1 (C10a), 163.8 (C2), 166.5 (CO), 176.9 (C5). LC-MS (ESI, 1.65 eV): m/z (\%) $400\left[100,(\mathrm{M}+\mathrm{Na}+\mathrm{MeOH})^{+}\right], 368\left[80,(\mathrm{M}+\mathrm{Na})^{+}\right], 346\left[15,(\mathrm{M}+\mathrm{H})^{+}\right]$. Anal. Calcd for $\mathrm{C}_{21} \mathrm{H}_{15} \mathrm{NO}_{4}$ (345.35): C, 73.03; H, 4.38; N, 4.06. Found: C, 72.94; H, 4.32; N, 4.11.

Ethyl 2,7-dimethyl-5-oxo-5H-chromeno[2,3-b]pyridine-3-carboxylate (5c). Yield: $0.116 \mathrm{~g}$ (78\%), yellow solid, mp $165-167{ }^{\circ} \mathrm{C}$ (ethanol); IR $\left(\mathrm{KBr}, v_{\max }, \mathrm{cm}^{-1}\right): 1724(\mathrm{C}=\mathrm{O}), 1668(\mathrm{C}=\mathrm{O}) .{ }^{1} \mathrm{H}$ 
NMR (300 MHz, $\mathrm{CDCl}_{3}$ ): $\delta 1.44\left(\mathrm{t}, J 7.2 \mathrm{~Hz}, 3 \mathrm{H}, 3-\mathrm{CH}_{2} \mathrm{CH}_{3}\right.$ ), 2.49 (s, 3H, 7-CH $\mathrm{CH}_{3}, 2.98$ (s, 3H, 2$\left.\mathrm{CH}_{3}\right), 4.44\left(\mathrm{q}, J 7.2 \mathrm{~Hz}, 2 \mathrm{H}, 3-\mathrm{CH}_{2} \mathrm{CH}_{3}\right), 7.51(\mathrm{~d}, J 8.5 \mathrm{~Hz}, 1 \mathrm{H}, 9-\mathrm{H}), 7.60(\mathrm{dd}, J 8.2,2.0 \mathrm{~Hz}, 1 \mathrm{H}, 8-$ $\mathrm{H}), 8.10(\mathrm{~d}, J 2.0 \mathrm{~Hz}, 1 \mathrm{H}, 6-\mathrm{H}), 9.20(\mathrm{~s}, 1 \mathrm{H}, 4-\mathrm{H}) .{ }^{13} \mathrm{C} \mathrm{NMR}\left(75 \mathrm{MHz}, \mathrm{CDCl}_{3}\right): \delta 14.3\left(3-\mathrm{CH}_{2} \mathrm{CH}_{3}\right)$, $20.9\left(7-\mathrm{CH}_{3}\right), 25.6\left(2-\mathrm{CH}_{3}\right), 61.7\left(3-\mathrm{CH}_{2} \mathrm{CH}_{3}\right), 114.0$ (C4a), 118.4 (C9), 121.3 (C5a), 123.4 (C3), 126.2 (C6), 135.2 (C7), 137.1 (C8), 140.6 (C4), 153.0 (C9a), 160.5 (C10a), 165.0 (3-CO), 166.6 (C2), 177.1 (C5). LC-MS (ESI, $1.65 \mathrm{eV}): \mathrm{m} / z(\%) 386\left[50,\left(\mathrm{M}+\mathrm{MeOH}+\mathrm{K}^{+} \mathrm{H}_{2} \mathrm{O}\right)^{+}\right], 352[100$, $\left.(\mathrm{M}+\mathrm{Na}+\mathrm{MeOH})^{+}\right], 330\left[10,(\mathrm{M}+\mathrm{MeOH}+\mathrm{H})^{+}\right], 298\left[37,(\mathrm{M}+\mathrm{H})^{+}\right]$, Anal. Calcd for $\mathrm{C}_{17} \mathrm{H}_{15} \mathrm{NO}_{4}$ (297.31): C, 68.68; H, 5.09; N, 4.71. Found: C, 68.77; H, 4.99; N, 4.60.

Ethyl 7-methyl-2-phenyl-5-oxo-5H-chromeno[2,3-b]pyridine-3-carboxylate (5d). Yield: $0.129 \mathrm{~g}$ (72\%), yellow solid, mp $174-176{ }^{\circ} \mathrm{C}$ (ethanol); IR $\left(\mathrm{KBr}, v_{\max }, \mathrm{cm}^{-1}\right): 1733(\mathrm{C}=\mathrm{O}), 1681(\mathrm{C}=\mathrm{O}) .{ }^{1} \mathrm{H}$ NMR (300 MHz, $\left.\mathrm{CDCl}_{3}\right): \delta 1.46\left(\mathrm{t}, J 7.2 \mathrm{~Hz}, 3 \mathrm{H}, 3-\mathrm{CH}_{2} \mathrm{CH}_{3}\right), 3.00\left(\mathrm{~s}, 3 \mathrm{H}, 7-\mathrm{CH}_{3}\right), 4.44$ (q, J 7.2 $\left.\mathrm{Hz}, 2 \mathrm{H}, 3-\mathrm{CH}_{2} \mathrm{CH}_{3}\right), 7.46-7.70(\mathrm{~m}, 7 \mathrm{H}, 2-\mathrm{Ph}, 8,9-\mathrm{H}), 8.11$ (s, 1H, 6-H), 9.10 (s, 1H, 4-H). ${ }^{13} \mathrm{C} \mathrm{NMR}$ $\left(75 \mathrm{MHz}, \mathrm{CDCl}_{3}\right): \delta 15.0\left(3-\mathrm{CH}_{2} \mathrm{CH}_{3}\right), 25.6\left(7-\mathrm{CH}_{3}\right), 61.8\left(3-\mathrm{CH}_{2} \mathrm{CH}_{3}\right), 113.7(\mathrm{C} 4 \mathrm{a}), 120.3(\mathrm{C} 9)$, 122.5 (C5a), 123.8 (C3), 126.1 (C6), 128.0 (C2',6'), 129.0 (C3',5'), 131.0 (C7), 133.0 (C4'), 135.9 (C8), 140.5 (C4), 140.6 (C1'), 153.8 (C9a), 160.1 (C10a), 164.7 (3-CO), 167.1 (C2), 175.9 (C5). LC-MS (ESI, $1.65 \mathrm{eV}): \mathrm{m} / z(\%) 414\left[100,(\mathrm{M}+\mathrm{Na}+\mathrm{MeOH})^{+}\right], 382\left[80,(\mathrm{M}+\mathrm{Na})^{+}\right], 360[15,(\mathrm{M}$ $+\mathrm{H})^{+}$]. Anal. Calcd for $\mathrm{C}_{22} \mathrm{H}_{17} \mathrm{NO}_{4}$ (359.37): C, 73.53; H, 4.77; N, 3.90. Found: C, 73.44; H, 4.66; $\mathrm{N}, 4.01$.

Ethyl 7-chloro-2-methyl-5-oxo-5H-chromeno[2,3-b]pyridine-3-carboxylate (5e). Yield: $0.122 \mathrm{~g}$ (77\%), yellow solid, mp 166-168 ${ }^{\circ} \mathrm{C}$ (ethanol); IR (KBr, $\left.v_{\max }, \mathrm{cm}^{-1}\right): 1741(\mathrm{C}=\mathrm{O}), 1659(\mathrm{C}=\mathrm{O}) .{ }^{1} \mathrm{H}$ NMR (300 MHz, $\mathrm{CDCl}_{3}$ ): $\delta 1.46\left(\mathrm{t}, J 7.2 \mathrm{~Hz}, 3 \mathrm{H}, 3-\mathrm{CH}_{2} \mathrm{CH}_{3}\right), 3.00\left(\mathrm{~s}, 3 \mathrm{H}, 2-\mathrm{CH}_{3}\right), 4.44$ (q, J 7.2 $\left.\mathrm{Hz}, 2 \mathrm{H}, 3-\mathrm{CH}_{2} \mathrm{CH}_{3}\right), 7.58(\mathrm{~d}, J 8.9 \mathrm{~Hz}, 1 \mathrm{H}, 9-\mathrm{H}), 7.74(\mathrm{dd}, J 8.9,2.5 \mathrm{~Hz}, 1 \mathrm{H}, 8-\mathrm{H}), 8.27(\mathrm{~d}, J 2.5$ $\mathrm{Hz}, 1 \mathrm{H}, 6-\mathrm{H}), 9.19$ (s, $1 \mathrm{H}, 4-\mathrm{H}) .{ }^{13} \mathrm{C} \mathrm{NMR}\left(75 \mathrm{MHz}, \mathrm{CDCl}_{3}\right): \delta 14.3\left(3-\mathrm{CH}_{2} \mathrm{CH}_{3}\right), 25.6\left(2-\mathrm{CH}_{3}\right)$, $61.8\left(3-\mathrm{CH}_{2} \mathrm{CH}_{3}\right.$ ), 113.7 (C4a), 120.3 (C9), 122.5 (C5a), 123.9 (C), 126.2 (C6), 131.1 (C7), 135.9 (C8), 140.6 (C4), 153.8 (C9a), 160.2 (C10a), 164.8 (3CO), 167.2 (C2), 175.9 (C5). LC-MS (ESI, $1.65 \mathrm{eV}): m / z(\%) 372 / 380\left[100,(\mathrm{M}+\mathrm{Na}+\mathrm{MeOH})^{+}\right], 356 / 358\left[20,(\mathrm{M}+\mathrm{K})^{+}\right], 318 / 320[80,(\mathrm{M}+$ $\mathrm{H})^{+}$]. Anal. Calcd for $\mathrm{C}_{16} \mathrm{H}_{12} \mathrm{ClNO}_{4}$ (317.72): C, 60.48; H, 3.81; N, 4.41. Found: C, 60.57; H, 3.90; N, 4.30 .

Ethyl 7-chloro-2-phenyl-5-oxo-5H-chromeno[2,3-b]pyridine-3-carboxylate (5f). Yield: $0.144 \mathrm{~g}$ (76\%), yellow solid, mp 167-169 ${ }^{\circ} \mathrm{C}$ (ethanol); IR (KBr, $\left.v_{\max }, \mathrm{cm}^{-1}\right)$ : $1736(\mathrm{C}=\mathrm{O}), 1685(\mathrm{C}=\mathrm{O}) .{ }^{1} \mathrm{H}$ NMR (300 MHz, $\mathrm{CDCl}_{3}$ ): $\delta 1.17\left(\mathrm{t}, J 7.1 \mathrm{~Hz}, 3 \mathrm{H}, 3-\mathrm{CH}_{2} \mathrm{CH}_{3}\right.$ ), 4.25 (q, $J 7.1 \mathrm{~Hz}, 2 \mathrm{H}, 3-\mathrm{CH}_{2} \mathrm{CH}_{3}$ ), 7.45-7.52 (m, 3H, 3',4',5'-H), $7.60(\mathrm{~d}, J 8.8 \mathrm{~Hz}, 1 \mathrm{H}, 9-\mathrm{H}), 7.63-7.68$ (m, 2H, 2',6'-H), 7.74 (dd, $J$ 8.8, $2.0 \mathrm{~Hz}, 1 \mathrm{H}, 8-\mathrm{H}), 8.30(\mathrm{~d}, J 2.0 \mathrm{~Hz}, 1 \mathrm{H}, 6-\mathrm{H}), 9.09$ (s, 1H, 4-H). ${ }^{13} \mathrm{C} \mathrm{NMR}\left(75 \mathrm{MHz}, \mathrm{CDCl}_{3}\right): \delta$ $13.8\left(3-\mathrm{CH}_{2} \mathrm{CH}_{3}\right), 62.0\left(3-\mathrm{CH}_{2} \mathrm{CH}_{3}\right), 114.2$ (C4a), 120.4 (C9), 122.8 (C3), 125.7 (C5a), 126.3 (C6), 128.4 (C2',6'), 129.0 (C3',5'), 130.1 (C7), 131.3 (C4'), 136.0 (C8), 138.4 (C1'), 140.3 (C4), 154.2 (C9a), 159.5 (C10a), 164.1 (3-CO), 166.4 (C2), 175.9 (C5). LC-MS (ESI, $1.65 \mathrm{eV}): \mathrm{m} / z$ (\%) 434/436 [100, $\left.(\mathrm{M}+\mathrm{Na}+\mathrm{MeOH})^{+}\right], 402 / 404\left[75,(\mathrm{M}+\mathrm{Na})^{+}\right], 380 / 382\left[15,(\mathrm{M}+\mathrm{H})^{+}\right]$. Anal. Calcd for $\mathrm{C}_{21} \mathrm{H}_{14} \mathrm{ClNO}_{4}$ (379.79): C, 66.41; H, 3.72; N, 3.69. Found: C, 66.34; H, 3.68; N, 3.78. 


\section{Acknowledgements}

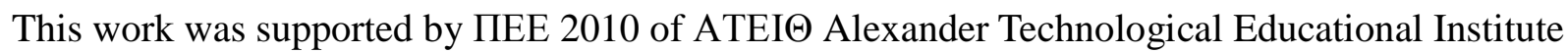
Research Fund (Project No: 2004.122.002.01).

\section{References}

1. Luche, J.-L. Synthetic Organic Sonochemistry; Plenum Press: New York, 1998.

2. Mason, T. J. Advances in Sonochemistry; JAI Press: London, 1990.

3. Cintas, P.; Luche, J.-L. Green Chem. 1999, 1, 115-125.

4. Puri, S.; Kaur, B.; Parmar, A.; Kumar, H. Curr. Org. Chem. 2013, 17, 1790-1828. http://dx.doi/10.2174/13852728113179990018

5. Cella, R.; Stefani, H. A. Tetrahedron 2009, 65, 2619-2641. http://dx.doi/10.1016/j.tet.2008.12.027

6. Roth, H. J.; Kleemann, A.; Beiswenger, T. Drug Synthesis, In Pharmaceutical Chemistry; Wiley: New York, 1988, Vol. 1.

7. Costantino, L.; Barlocco, D. Curr. Med. Chem. 2006, 13, 65-85. http://dx.doi/10.2174/092986706775197999

8. Muegge, I. Med. Res. Rev. 2003, 23, 302-321. http://dx.doi/10.1002/med.10041

9. Hegab, M. I.; Abdel-Fattah, A.-S. M.; Yousef, N. M.; Nour, H. F.; Mostafa, A. M.; Ellithey, M. Arch. Pharm. Chem. Life Sci. 2007, 340, 396-403. http://dx.doi/10.1002/ardp.200700089

10. Unangst, P. C.; Capiris, T.; Connor, D. T.; Heffner, T. G.; Mackenzie, R. G.; Miller, S. R.; Pugsley, T. A.; Wise, L. D. J. Med. Chem. 1997, 40, 2688-2693. http://dx.doi./10.1021/jm970170v

11. Giannouli, V.; Kostakis, I. K.; Pouli, N.; Marakos, P.; Kousidou, O. C.; Tzanakakis, G. N.; Karamanos, N. K. J. Med. Chem. 2007, 50, 1716-1719. http://dx.doi./10.1021/jm061410m

12. For recent reviews, see:

(a) Moreau, J.; Hurvois, J.-P.; Mbaye, M. D.; Renaud, J.-L. In: Targets in Heterocyclic Systems, Attanasi, O. A; Spinelli, D. Eds.; Italian Society of Chemistry, Rome, 2009; Vol. 13, pp. 201230.

(b) Tanaka, H.; Namekata, I.; Komikado, C.; Kawanishi, T.; Shigenobu, K. Curr. Top. Pharmacol. 2007, 11, 1-15.

(c) Quirion, J.-C.; Leclerc, E.; Jubault, P. Product subclass 18: 1,2-dihydropyridines, 1,4dihydropyridines, and derivatives. In Science of Synthesis, Thieme Verlag, Stuttgart, 2007, Vol. 33 , pp 601-628.

(d) Lavilla, R. Curr. Org. Chem. 2004, 8, 715-737. http://dx.doi/10.2174/1385272043370690

(e) Lavilla, R. J. Chem. Soc., Perkin Trans. 1 2002, 1141-1156. 
13. Meyer, M. D.; Altenbach, R. J.; Basha, F. Z.; Carroll, W.A.; Drizin, I.; Kerwin, J. F. Jr.; Wendt, M. D.; Haight, A. R.; Zhang, W. U.S. Patent 6046 207, April, 4, 2000.

14. Brown, R. E.; Puchalski, C.; Shavel, J. Jr. U.S. Patent 3962 266, May, 1, 1975.

15. Connor, D. T.; Unangst, P. C.; Schwender, C. F.; Sorenson, R. J.; Carethers, M. E.; Puchalski, C.; Brown, R. E. J. Heterocyclic Chem. 1984, 21, 1561-1564.

16. (a) Zarganes-Tzitzikas, T.; Terzidis, M. A.; Stephanidou-Stephanatou, J.; Tsoleridis, C. A.; Kostakis G. E. J. Org. Chem. 2011, 76, 9008-9014. http://dx.doi/10.1021/jo201732g

(b) Terzidis, M. A.; Zarganes-Tzitzikas, T.; Tsimenidis, C.; Stephanidou-Stephanatou, J.; Tsoleridis, C. A.; Kostakis G.E. J. Org. Chem. 2012, 77, 9018-9028. http://dx.doi/10.1021/j03014947

17. Dimitriadou, E.; Stephanidou-Stephanatou, J.; Tsoleridis, C. A.; Hadjipavlou-Litina, D. J.; Kontogiorgis, C.; Kostakis, G. E. Tetrahedron 2014, 70, 2938-2943. http://dx.doi.org/10.1016/j.tet.2014.03.031

18. Ghosh, C. K; SinhaRoy, D. K.; Mukhopadhyay, K. K. J. Chem. Soc., Perkin Trans. 1 1979, 1964-1968.

19. Ghosh, C. K. Synth. Comm. 1978, 487-490.

20. Kubo, K.; Ukawa, K.; Kuzuna, S.; Nohara, A. Chem. Pharm. Bull. 1986, 1108-1117.

21. Ibrahim, M.A. Synth. Commun. 2009, 39, 3527-3545. http://dx.doi./10.1080/00397910902788141

22. Hadjipavlou-Litina, D.; Magoulas, G. E.; Bariamis, S. E.; Drainas, D.; Avgoustakis, K.; Papaioannou, D. Bioorg. Med. Chem. 2010, 18, 8204-8217. http://dx.doi.org/10.1016/j.bmc.2010.10.012

23. Neochoritis, C. G.; Tsoleridis, C. A.; Stephanidou-Stephanatou, J.; Kontogiorgis, C. A.; Hadjipavlou-Litina, D. J. J. Med. Chem. 2010, 53, 8409-8420. http://dx.doi./10.1021/jm100739n 